\title{
Synthesis of carbon nanotubes loaded hydroxyapatite: Potential for controlled drug release from bone implants
}

\author{
Lisa COSTANTINI $^{a}$, Nikolaos BOUROPOULOS ${ }^{b, c}$, Dimitrios G. FATOUROS $^{d}$, \\ Ioanna KONTOPOULOU ${ }^{b}$, Marta ROLDO ${ }^{a, *}$ \\ ${ }^{a}$ School of Pharmacy and Biomedical Science, University of Portsmouth, St Michael's Building, \\ White Swan Road, Portsmouth, UK \\ ${ }^{b}$ Department of Materials Science, University of Patras, 26504 Rio, Patras, Greece \\ ${ }^{c}$ Foundation for Research and Technology, Hellas-Institute of Chemical Engineering and High Temperature \\ Chemical Processes-FORTH/ICEHT, P.O. Box 1414, GR 26504 Patras, Greece \\ ${ }^{d}$ Department of Pharmaceutical Technology, School of Pharmacy, Aristotle University of Thessaloniki, \\ GR-54124 Thessaloniki, Greece
}

Received: March 02, 2016; Revised: June 10, 2016; Accepted: June 11, 2016

(C) The Author(s) 2016. This article is published with open access at Springerlink.com

\begin{abstract}
Carbon nanotubes (CNTs) can support the building of flexible and porous scaffolds for bone regeneration. Various studies have looked at the mixing of CNTs with hydroxyapaptite for the formulation of bone implants. In the present work, we report the one step preparation and characterisation of chitosan/hydroxyapatite/CNTs composite materials obtained by wet precipitation of hydroxyapatite (HA) in the presence of chitosan or its amphiphilic derivative $\mathrm{N}$-octyl- $\mathrm{O}$-sulphate chitosan and CNTs. The in situ precipitation of HA assured inclusion of the polysaccharide and the CNTs in the HA structure and provided materials with the ability to control the release of different model drugs.
\end{abstract}

Keywords: carbon nanotubes (CNTs); hydroxyapatite (HA) composites; bone regeneration; chitosan

\section{Introduction}

Tissue engineering research has recently focused, amongst other materials, on the use of carbon nanotubes (CNTs) in synthetic scaffolds [1]. CNTs are allotropes of carbon, described as rolled up graphene sheets composed of $\mathrm{sp}^{2}$ carbon. Depending on the number of graphene sheet concentrically rolled up to form the tube, single-wall nanotubes (SWNTs) or multi-wall nanotubes (MWNTs) can be obtained [2]; their characteristics and main biomedical applications have

\footnotetext{
* Corresponding author.

E-mail: marta.roldo@port.ac.uk
}

been recently reviewed [3]. Carbon nanotubes are of interest in tissue engineering because they can support the building of flexible and porous structures similar to the extracellular matrix (ECM), environment in which cells physiologically migrate and proliferate to form tissues and organs [4]. Bone regeneration is one of the fields where the inclusion of CNTs in synthetic structures is of particular interest. Natural bone includes both inorganic (hydroxyapatite, HA) and organic (collagen) components; its complex structure can be simulated by synthetic HA/polymer composites. Researchers have investigated the use of polymers and polymeric hydrogels as matrices forming materials for this application, and interest has recently been focused 
on carbon nanotubes as a material that allows to strengthen and increase the roughness and porosity of these matrices [5]. Interestingly, $\mathrm{Li}$ et al. [6] demonstrated that MWNTs are able to induce ectopic bone formation in vivo by stimulating the differentiation of inducible cells from soft tissue to osteogenic cells, further supporting the potential of these carbon nanomaterials in bone regeneration applications. Amongst the polymers studied for the formation of composite structures, the following hydrophobic materials have been considered: $\operatorname{poly}(L$-lactic acid) (PLLA) [7,8], poly(caprolactone) (PCL) [9], and poly(methyl methacrylate) (PMMA) [10]. In most cases, the use of hydrophobic polymers limits cell migration, and the introduction of hydrophilic groups on the surface of CNTs, such as carboxylic moieties, is necessary to improve the performance of these materials. As an alternative, hydrophilic polymers have been considered. Scaffolds containing the hydrophilic semi-synthetic polysaccharide chitosan have been studied. Chitosan and its derivatives have been extensively used as materials for the fabrication of hydrogels for tissue engineering applications [11]; however, the polysaccharide does not present osteoconductive properties on its own. This problem can be overcome by the addition of HA to enhance bioactivity. Also in this case, addition of CNTs can further improve the mechanical performance. Such materials, as prepared by Im et al. [12], present good cell adhesion due to the CNTs' contribution to the enhancement of the nanoroughness of the chitosan scaffolds; they were obtained by simple mixing of HA and CNTs in chitosan solution followed by freeze drying. Similarly, Fonseca-García et al. [13] prepared chitosan/HA/CNTs scaffolds containing oxygen and nitrogen doped CNTs. They freezed the polymeric suspension of HA and CNTs and ejected it from a syringe at a constant speed, achieving the formation of well-aligned micro-channel structures. Lu et al. [14], on the other hand, produced foam like chitosan/HA scaffolds in which CNTs were grown in situ by a chemical vapour deposition method; they obtained highly crystalline nanotubes in $\mathrm{Ca}$ deficient $\mathrm{HA}$ matrices. The matrices present interesting superparamagnetic properties claimed to be potentially responsible for the attraction of growth factors, stem cells, and other bioactive molecules. Further work needs to be done in order to prove these claims.

Works so far published relied on the physical mixture of CNTs with preformed HA, while, in the present work, we report the facile, one step preparation of chitosan/HA/CNTs materials obtained by an HA wet precipitation method in the presence of chitosan or its amphiphilic derivative $N$-octyl- $O$-sulphate chitosan and CNTs for a more homogeneous interaction among the three composite components. The materials obtained by in situ precipitation were characterised by dynamic vapour sorption (DVS), X-ray diffraction (XRD), and thermal gravimetric analysis (TGA), and further formulated into compressed scaffolds from which the release of model drugs was studied.

\section{Materials and methods}

\section{1 Materials}

Low viscosity chitosan, ibuprofen, ibuprofen sodium salt, fluorescein isothiocyanate-dextran, tricalcium phosphate (TCP), and methylcellulose were obtained from Sigma-Aldrich (Poole, UK). All other solvents and inorganic salts were purchased from Fisher Scientific, Loughborough, UK. Carboxylated single-wall (outer diameter $1-4 \mathrm{~nm}$, length 3-30 $\mu \mathrm{m}$, $-\mathrm{COOH}$ content $7 \pm 1.5 \mathrm{wt} \%$ ) and multi-wall (outer diameter $8-15 \mathrm{~nm}$, length $10-50 \mu \mathrm{m},-\mathrm{COOH}$ content $2.56 \mathrm{wt} \%$ ) carbon nanotubes were obtained from Cheaptubes Ltd., USA. $\mathrm{N}$-octyl- $\mathrm{O}$-sulphate chitosan (NOSC) was prepared as described previously $[15,16]$.

\section{2 Synthesis of HA composites}

Hydroxyapatatite was prepared by co-precipitation of $\mathrm{CaCl}_{2}$ and $\mathrm{NaH}_{2} \mathrm{PO}_{4}$ according to the method described by Tanase et al. [17,18] with modification. Briefly, $\mathrm{CaCl}_{2}(12.7 \mathrm{~mL}, 53 \% \mathrm{w} / \mathrm{v})$ and $\mathrm{NaH}_{2} \mathrm{PO}_{4}(13.3 \mathrm{~mL}$, $32.5 \% w / v)$, with $\mathrm{Ca} / \mathrm{P}$ ratio $1: 1.67$, were slowly added to $100 \mathrm{~mL}$ medium composed as shown in Table 1 . The $\mathrm{pH}$ was adjusted to 7.0 by slow and controlled addition of $\mathrm{NH}_{4} \mathrm{OH}(25 \% \mathrm{w} / \mathrm{v})$. The reaction mixture was

Table 1 Composition of the medium employed in the synthesis of HA composites and controls. The polymer:HA ratio was maintained at 30:70

\begin{tabular}{lccc}
\hline Composite & Solvent & Polymer & CNTs $^{*}(20 \mathrm{mg})$ \\
\hline HA control $\left(\mathrm{H}_{2} \mathrm{O}\right)$ & $\mathrm{H}_{2} \mathrm{O}$ & - & - \\
HA-NOSC & $\mathrm{H}_{2} \mathrm{O}$ & NOSC & - \\
HA-NOSC-SWNTs & $\mathrm{H}_{2} \mathrm{O}$ & NOSC & SWNTs \\
HA-NOSC-MWNTs & $\mathrm{H}_{2} \mathrm{O}$ & NOSC & MWNTs \\
HA control $(\mathrm{HCl})$ & $\mathrm{HCl} 1.5 \% v / v$ & - & - \\
HA-CS & $\mathrm{HCl} 1.5 \% v / v$ & Chitosan & - \\
HA-CS-SWNTs & $\mathrm{HCl} 1.5 \% v / v$ & Chitosan & SWNTs \\
HA-CS-MWNTs & $\mathrm{HCl} 1.5 \% v / v$ & Chitosan & MWNTs \\
\hline *CNTs were dispersed in polymer solution by sonication for 180 min.
\end{tabular}


magnetically stirred at $37^{\circ} \mathrm{C}$ for 7 days, and the $\mathrm{pH}$ was daily checked and adjusted as required. The precipitate was finally washed with deionised water and collected by centrifugation ( $2 \mathrm{~min}$ at $2000 \mathrm{rpm}$ ); this process was repeated until the conductivity of the supernatant was reduced to that of deionised water. The samples were then dried under vacuum at $60{ }^{\circ} \mathrm{C}$ overnight.

\section{3 Characterisation of HA composites}

Dynamic vapour sorption (DVS) analysis was carried out with Surface Measurement Systems DVS Advantage using nitrogen as the carrier gas; the mass change of samples subjected to a changing vapour partial pressure at $25^{\circ} \mathrm{C}$, using either water or octane, was recorded. Water partial pressure was increased from 0 to $90 \%$ in $10 \%$ increment; while octane partial pressure was increased from 0 to $10 \%$ in $1 \%$ increment and from $10 \%$ to $90 \%$ in $10 \%$ increment, and a final step to $95 \%$ was introduced. In both cases a full adsorption/desorption cycle was performed. The data collected were used to calculate the water adsorption isotherms and the total water absorbed per sample. The data collected with octane were used to calculate the total pore volume according to

$$
\begin{aligned}
& \text { Total pore volume }\left(\mathrm{cm}^{3} / \mathrm{g}\right)= \\
& \frac{\text { Total octane absorbed }(\mathrm{g})}{0.703\left(\mathrm{~g} / \mathrm{cm}^{3}\right) \times \text { sample mass }(\mathrm{g})}
\end{aligned}
$$

Composites were further characterised by means of attenuated total reflectance (ATR) spectroscopy; spectra were recorded on a Varian 640-IR spectrophotometer (Palo Alto, CA, USA). The surface morphology of the materials and the inclusion of CNTs in the composite materials were investigated with a Jeol JSM-6160L scanning electron microscope with electron backscatter and energy dispersive X-ray (EDX) spectroscopy capability (Silicon Drift Detector (SDD)-X-MaxN, Oxford Instruments); EDX spectroscopy provided quantitative information about the presence of $\mathrm{Ca}, \mathrm{P}$, and $\mathrm{Cl}$ ions on the surface of the samples analysed. Samples were sputter coated with gold at low vacuum in a Polaron e500 provided by Quoram Technologies. Powder X-ray diffraction (XRD) spectra were acquired using a standard powder diffractometer (Siemens D8) with $\mathrm{Ni}$-filtered $\mathrm{Cu} \mathrm{K} \alpha_{1}$ radiation $(\lambda=0.154059 \mathrm{~nm})$. Thermal gravimetric analysis (TGA) measurements were carried out with a TGA Q500 from TA Instruments, in the temperature range between room temperature and $600{ }^{\circ} \mathrm{C}$, with a heating rate of $10{ }^{\circ} \mathrm{C} / \mathrm{min}$ in nitrogen atmosphere.

\section{4 Formulation of HA composite scaffolds and model drug loading}

HA controls and composites were formulated into compressed form as previously described by Green et $a l$. [15]. Ibuprofen (IBU), ibuprofen sodium (IBU-Na), and fluorescein isothiocyanate-dextran (FITC-Dex, $40 \mathrm{kDa}$ ) were used as model drugs. Ibuprofen and ibuprofen sodium were added to the compressed materials in a $20 \% w / w$ ratio. The HA or HA composite/ TCP ratio was kept constant at 25:75, while the ratio of polymer/methylcellulose was kept at 80:20, and together these polysaccharides contributed to $20 \%$ of the total mass (Table 2). Drug loading was carried out by adsorption; the HA material was mixed with IBU-Na or IBU in a mortar and soaked with $400 \mu \mathrm{L}$ deionised water or $100 \mu \mathrm{L}$ ethanol, respectively. A paste was obtained and dried at $60{ }^{\circ} \mathrm{C}$ for half an hour under vacuum; then all other components were added, soaked again with water, and dried in the oven for another hour in order to obtain dry powder. $200 \mathrm{mg}$ tablets were obtained by pressing the powder for $5 \mathrm{~min}$ at 3.5 tons. The same method was followed in the preparation of FITC-Dex tablets.

\section{5 Physical characterisation of the compressed scaffolds}

Scaffolds' hardness and friability were tested. Hardness was measured as the resistance of the tablet to crushing when compressed between two jaws facing each other. The two jaws were drawn together by applying a force and the total force needed to crush the tablet was recorded. All experiments were carried out in triplicate. Friability was measured by introducing three tablets of each type in a drum as described by the British Pharmacopoeia (BP). Friability, expressed as the percentage of weight loss, was calculated by weighing the tablets after $0,25,50,75$, and 100 rotations of the drum. The drum was rotated so that the tablets were exposed to 100 falls in $4 \mathrm{~min}$. Disintegration of the tablets exposed to buffer was also observed. This test was conducted using the disintegration apparatus, as

Table 2 Composition of the compressed scaffolds

\begin{tabular}{lcc}
\hline & IBU or IBU-Na & FITC-Dex \\
\hline HA or HA composite (mg) & 144 & 178 \\
TCP ( $\beta$-tricalcium phosphate) (mg) & 432 & 534 \\
Chitosan (mg) & 115 & 142 \\
Methylcellulose (mg) & 29 & 36 \\
Drug (mg) & 180 & 10 \\
\hline
\end{tabular}


described by the BP. The samples were immersed in a vessel containing PBS buffer at $37 \pm 2{ }^{\circ} \mathrm{C}$ and stirred at 29-32 cycles/min for $15 \mathrm{~min}$.

\section{6 Drug release studies}

Release studies were carried out in a standard paddle dissolution apparatus. Tablets were immersed in 500 $\mathrm{mL}$ phosphate buffer at $\mathrm{pH}=7$ and incubated at $37^{\circ} \mathrm{C}$ under continuous stirring. At pre-set time, $5 \mathrm{~mL}$ solution was sampled and replaced with fresh buffer equilibrated at $37{ }^{\circ} \mathrm{C}$. The release of FITC-Dex was carried out in amber vessel. The concentration of IBU and IBU-Na in the release medium was tested by measuring the UV absorbance at $224 \mathrm{~nm}$ (UV Thermo Scientific Nicolet Evolution 10), while the release of FITC-Dex was studied by fluorescent spectroscopy (excitation wavelength $494 \mathrm{~nm}$, emission wavelength $518 \mathrm{~nm}$, Varian Cary Eclipse Fluorescence Spectrophotometer). In order to determine the total amount of drug loaded in each sample, the total drug release from crushed tablets dispersed in $500 \mathrm{~mL}$ buffer, subjected to sonication for $1 \mathrm{~h}$, and incubated at $37{ }^{\circ} \mathrm{C}$ for $48 \mathrm{~h}$, was determined. All experiments were conducted in triplicate.

\section{Results and discussion}

\section{1 Synthesis and characterisation of HA composites}

HA and its composite derivatives were obtained as compact materials that were further grinded into powder. The average amount of material obtained from the reaction was $6.61 \pm 0.02 \mathrm{~g}(n=6)$. During the washing step, it was observed that the ionic strength of the washing medium used for HA prepared in water and in presence of NOSC remained high even after several washes $\left(0.28\right.$ and $0.23 \mathrm{mS} \cdot \mathrm{cm}^{-1}$, respectively, as compared to the value of deionised water, $\left.\sim 20-30 \mu \mathrm{S} \cdot \mathrm{cm}^{-1}\right)$. The infrared analysis of the composites confirmed the formation of HA (Fig. 1(a)) with characteristic $\mathrm{PO}^{3-}$ and $\mathrm{PO}^{4-}$ vibrations at 560,600 , and $1007 \mathrm{~cm}^{-1}$, and $\mathrm{OH}^{-}$stretching mode at $3413 \mathrm{~cm}^{-1}$ (not shown). Peaks at 1633 and $1456 \mathrm{~cm}^{-1}$ (not shown) were assigned to the presence of $\mathrm{CO}_{3}^{2-}$ groups that might have been included due to reaction of atmospheric $\mathrm{CO}_{2}[15,19]$; these peaks were not observed in $\mathrm{HA}$ prepared in the presence of $\mathrm{HCl}$, possibly due to the reduced solubility of $\mathrm{CO}_{2}$ in water at acidic $\mathrm{pH}$ [19]. Infrared spectra of composites did not show characteristic peaks of chitosan or NOSC, due to an overlapping effect of the HA bands [20]. This was also confirmed by recording the ATR spectra of physical mixtures of HA and chitosan containing from $1 \%$ to $30 \%$ of chitosan; in all cases the characteristic peaks of chitosan were not visible (data not shown). The crystallinity index calculated from the infrared data, using a method previously described [21,22] (Table 3), showed that the control HA and the HA prepared in the presence of CNTs had similar crystallinity to fresh bone and enamel, as reported by Reyes-Gasga et al. [23]; however, the presence of only polymer induced a decrease in the crystallinity index. XRD patterns of the composites (Figs. 1(b) and 1(c)) showed peaks typical of both CNTs and HA. The XRD patterns of MWNTs and SWNTs exhibited characteristic peaks at about $26^{\circ}$ and $44^{\circ}$ which correspond to (002) and (100) diffraction planes of the hexagonal graphite structure $[24,25]$. XRD patterns showed also that there is no evidence of the formation of calcium phosphate compounds other than HA. The patterns showed the characteristic peak at $25.9^{\circ}$ indexed to the (002) reflection of HA which however overlapped with the (002) reflection of carbon
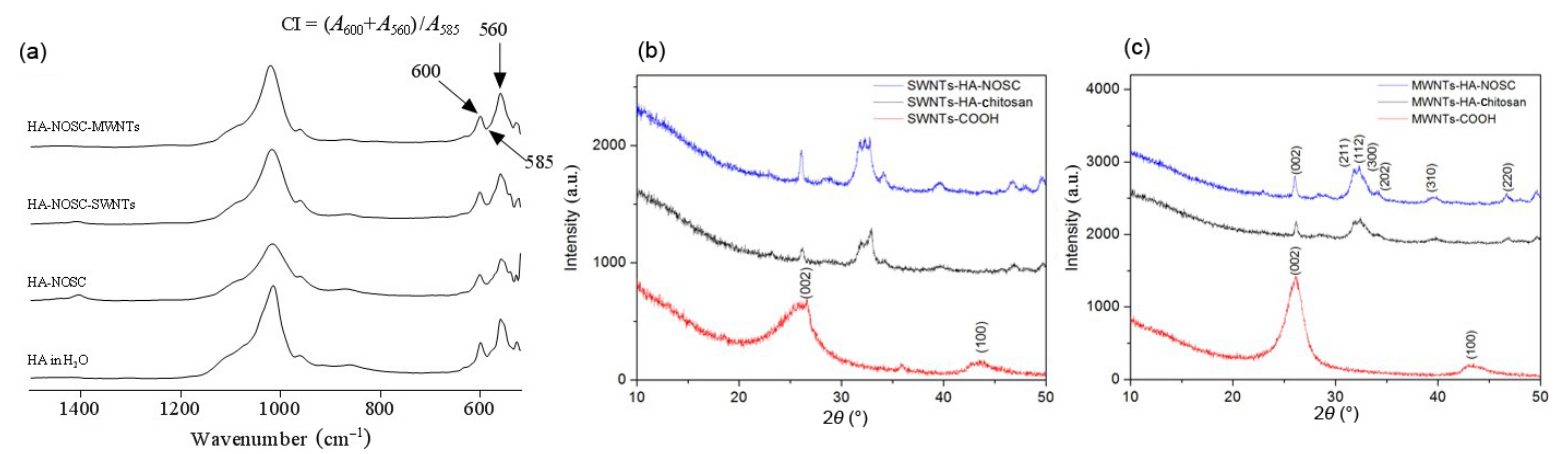

Fig. 1 (a) Infrared absorbance spectra of HA and its composites containing NOSC and CNTs. The graph also shows how the infrared crystallinity index (CI, Table 3) has been calculated according to the height method suggested by Weiner and Bar-Yosef. (b) and (c) XRD analysis of SWNTs and MWNTs containing composites, respectively. 
Table 3 Infrared derived crystallinity index

\begin{tabular}{lcc}
\hline & Chitosan & NOSC \\
\hline HA & 2.56 & 2.82 \\
HA-polymer & 1.96 & 1.58 \\
HA-polymer-SWNTs & 2.07 & 2.21 \\
HA-polymer-MWNTs & 2.05 & 2.17 \\
\hline
\end{tabular}

nanotubes. Furthermore, the reflections at $31.7^{\circ}, 32.2^{\circ}$, and $32.9^{\circ}$ respectively corresponding to the (211), (112), and (300) reflections were overlapped indicating low crystallinity of HA and the presence of nanocrystals [26]. The morphological characterisation of the composite materials was carried out by scanning electron microscopy (SEM), and the images were also used to confirm the inclusion of CNTs in the structures. Figure 2 shows the morphology of CNTs as received. These are present as bundles of entangled tubes; therefore, before inclusion, CNTs were suspended in a solution of NOSC or chitosan by sonication, process known to stabilise and separate the CNTs into single entities [16,27,28].

Figure 3(a) shows the presence of some crystalline impurities in the HA control prepared in water; this is consistent with the earlier observation of the high conductivity measured in the washing medium, further confirmed by the high water absorption observed in the DVS study (see below). This was not the case for the HA control sample prepared in $\mathrm{HCl}$ (Fig. 3(b)) which displayed a lower $\mathrm{Cl} / \mathrm{Ca}$ ratio (0.02) and a higher $\mathrm{Ca} / \mathrm{P}$ ratio (1.35) than the HA control prepared in water $(0.04$ for $\mathrm{Cl} / \mathrm{Ca}$ and 1.16 for $\mathrm{Ca} / \mathrm{P})$. It is hypothesised that
$\mathrm{CaCl}_{2}$ in water is able to dissociate immediately and start reacting with the $\mathrm{PO}_{4}^{3-}$ before addition of the base leading to formation of $\mathrm{CaHPO}_{4}$ crystals, while in the presence of $\mathrm{HCl}$, due to the common ion effect, the dissociation of $\mathrm{CaCl}_{2}$ does not occur until the $\mathrm{pH}$ increases and more of the calcium is involved in the formation of HA. A similar phenomenon was observed in the composite prepared in the presence of NOSC (Fig. $3(\mathrm{c})$ ); in this case, dissociation of $\mathrm{CaCl}_{2}$ in the starting solution would be further favoured by interactions of $\mathrm{Ca}^{2+}$ with the sulphate groups of NOSC. SEM images (Figs. 3(e)-3(h)) of the composites including CNTs showed that only few of the carbon nanostructures were visible on the surface of the composites. This is attributed to the fact that CNTs are coated with the polymers and HA is grown on their surface; therefore the nanotubes are successfully embedded in the materials. Nevertheless, few CNTs were visible mainly near fracture sites of the materials. In a study conducted by Orsmby et al. [10], it was possible to observe a similar distribution with consequent reinforcement of the craze by CNTs bridging sub-micron voids and preventing pore coalescence.

The $\mathrm{Ca} / \mathrm{P}$ ratio of the composite material surfaces was obtained by energy dispersive analysis (Table 4). The $\mathrm{Ca} / \mathrm{P}$ ratio in $\mathrm{HA}$ is 1.67 . Due to preparation at physiological $\mathrm{pH}$, a lower ratio was expected [19]. The lower ratio obtained for the composites prepared in this study could be explained by the fact that phosphorus might be present as other phosphate salts as well as in
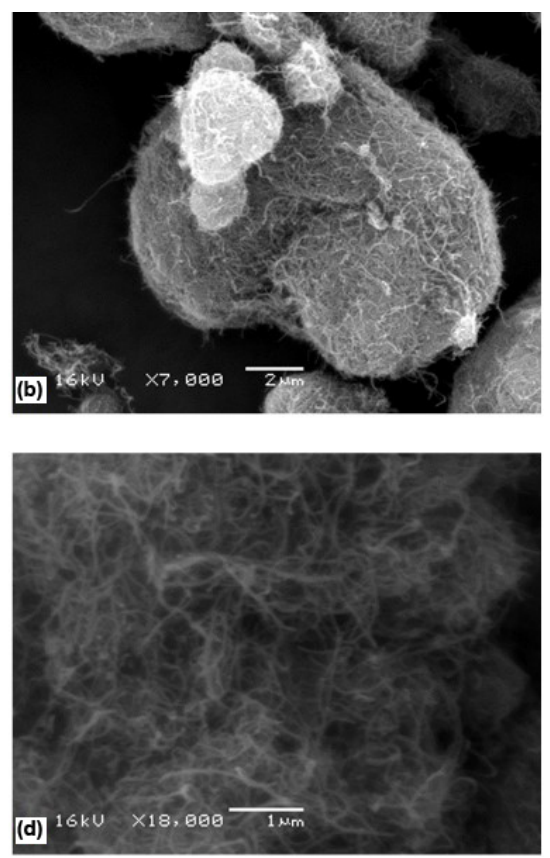

Fig. 2 SEM images of (a, c) SWNTs-COOH and (b, d) MWNTs-COOH as received. 

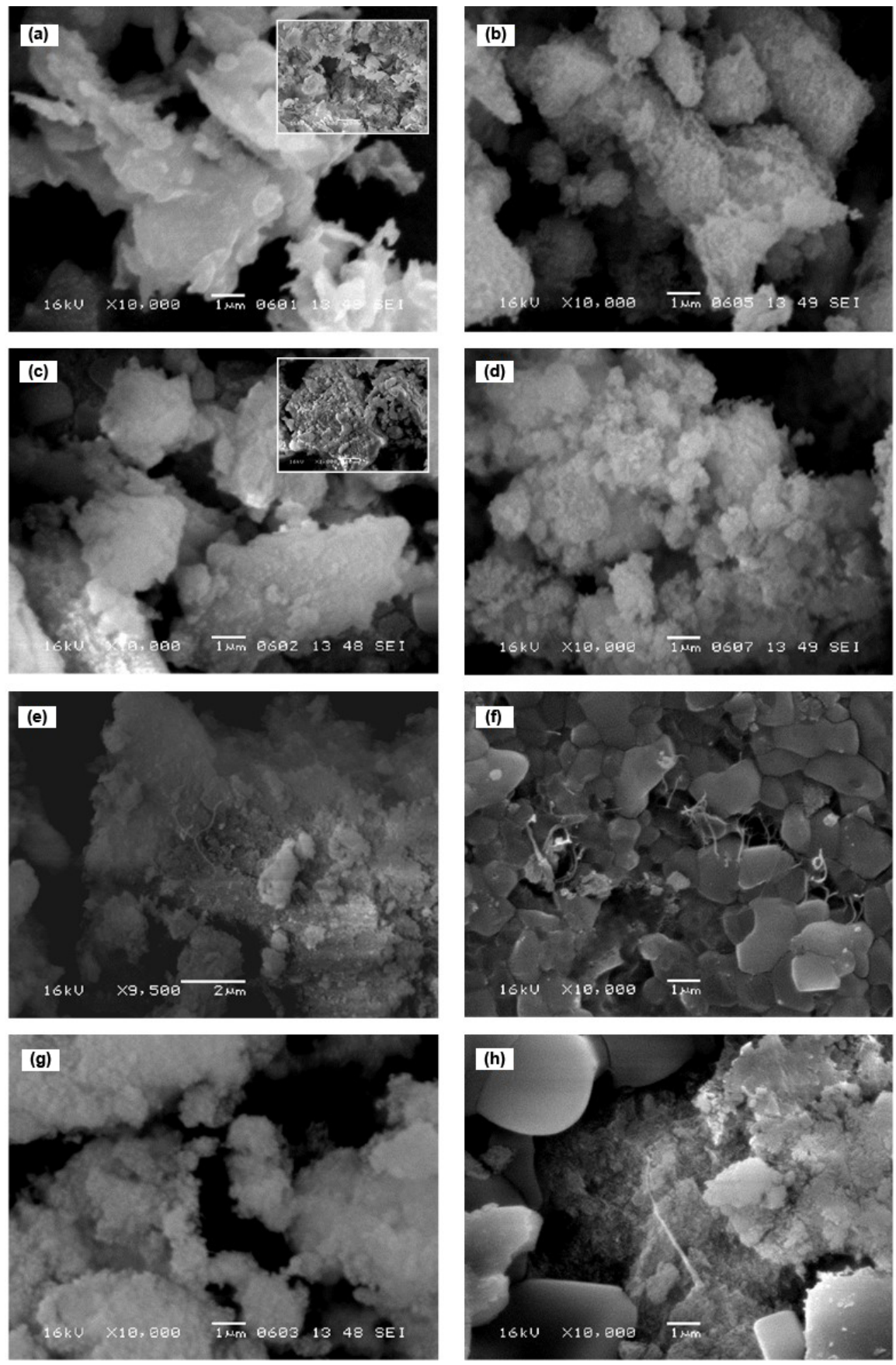

Fig. 3 SEM images of HA composites: (a) HA control in water, (b) HA control in HCl, (c) HA-NOSC, (d) HA-CS, (e) HA-NOSC-SWNTs, (f) HA-CS-SWNTS, (g) HA-NOSC-MWNTs, (h) HA-CS-MWNTs.

Table 4 Surface $\mathrm{Ca} / \mathrm{P}$ ratio in different $\mathrm{HA}$ composite materials

\begin{tabular}{lcc}
\hline & Chitosan & NOSC \\
\hline HA & 1.34 & 1.16 \\
HA-polymer & 1.38 & 1.13 \\
HA-polymer-SWNTs & 1.25 & 1.32 \\
HA-polymer-MWNTs & 1.23 & 1.22 \\
\hline
\end{tabular}

HA. This is also reflected by the fact that HA prepared in water and HA-NOSC showed evidence of crystalline salts tightly associated with the materials.

The water uptake of the material has an effect on the release by diffusion of loaded drugs as well as on the rate of degradation of the material [29]. Therefore, dynamic sorption of water vapour on the composite materials was studied; samples were subjected to varying vapour concentrations at a controlled temperature, and the changes in mass were recorded over time (Fig. 4). All samples presented a type III 

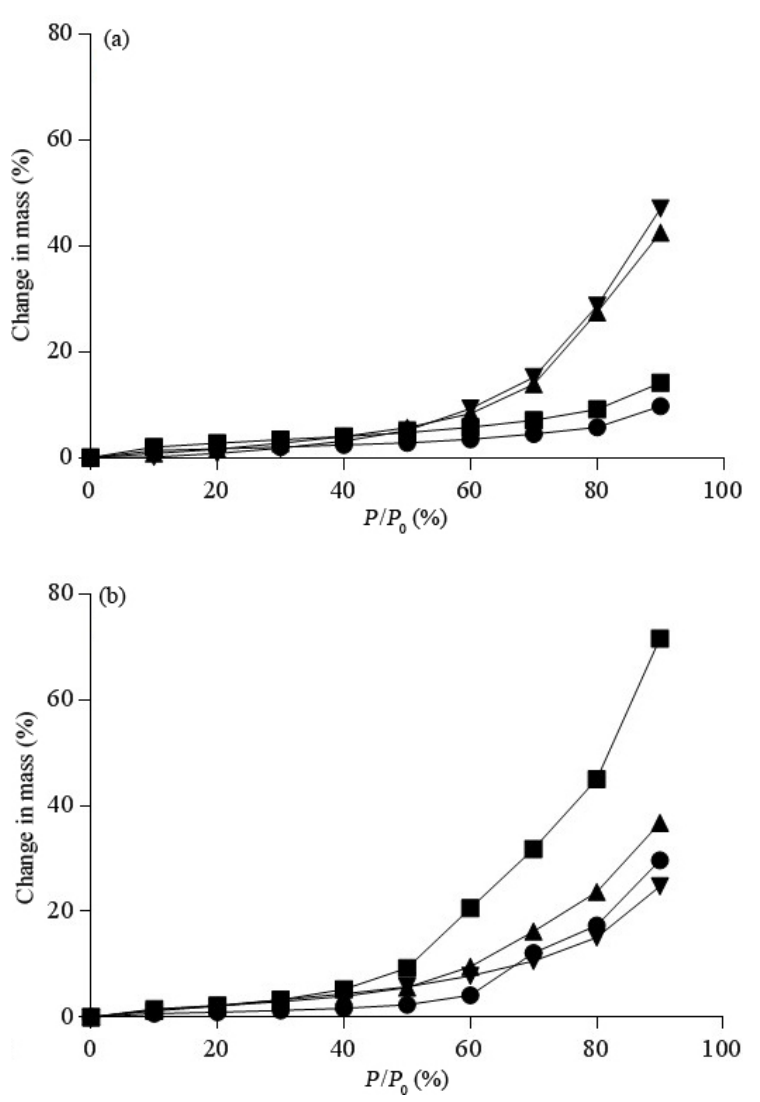

Fig. 4 Water sorption isotherm profiles for HA ( $-\mathbf{-})$, HA-polymer $(\boldsymbol{-})$, HA-polymer-SWNTs $(\boldsymbol{-}-)$, HA-polymer-MWNTs $(\nabla-)$. The polymer in (a) is chitosan and the polymer in (b) is NOSC.

isotherm, indicative of cluster mechanism in water sorption as opposed to monolayer formation. The water sorption analysis further confirmed that the initial $\mathrm{pH}$ and composition of the solution employed in the synthesis of HA affected the characteristics of the final product. HA prepared in water achieved a total water absorption of $29.7 \%$ as compared to HA prepared in an initial acidic solution that absorbed $9.8 \%$ of water. This, together with the SEM images, confirmed that hydrophilic salts had formed and deposited within the composite when the $\mathrm{pH}$ of the initial medium was neutral, while an initial acidic $\mathrm{pH}$ did not favour the formation of these crystals. The addition of chitosan to the composites led to an increase of $4.4 \%$ in total sorbed water, while the addition of NOSC had a more evident effect with an increase of $42 \%$. The more crystalline chitosan might work more as an absorption barrier and limit the amount of water able to penetrate in the composite structure despite its hydrophilicity; on the other hand, the amorphous NOSC does not constitute such a barrier and favours water absorption [15]. Furthermore, chitosan solubility is known to be limited in water while NOSC tends to be freely soluble, therefore recalling more water than the parent polymer. Finally, it was postulated that the $\mathrm{Ca}^{2+}$ ions would interact with the hydroxylic groups of chitosan giving rise to a tightly bound complex between the polysaccharide and the forming HA [17], leaving less hydrophilic groups available for interaction with water. The addition of CNTs in the composite structure affected in a different manner composites containing chitosan and NOSC. In the former case, the introduction of the carbon nanostructures induced an increase in sorbed water. This could be explained by the fact that the nanotubes affected the spatial arrangement of the polysaccharide and induced the formation of a structure with different porosity. On the other hand, the hydrophobicity of CNTs seems to have a more direct effect on water sorption in composites containing NOSC where the total amount of sorbed water decreases after addition of SWNTs and MWNTs. TGA thermographs showed a lower total degradation of composites containing NOSC compared to composites containing chitosan, indicating that a higher concentration of CNTs was included when the amphiphilic polysaccharide was used, further justifying the observed increased hydrophobicity of the composites (Fig. 5).

Dynamic sorption of octane was used to calculate the total pore volume (Fig. 6) and further explain these findings. In the case of composites containing chitosan, the control and HA-CS presented very similar total pore volume correlated with very similar total water sorbed. A decrease in total pore volume was observed after CNTs inclusion in the composite; however, this corresponded to an increase in total water sorbed, indicating that possibly bulk water diffusion is taking place rather than just pore diffusion. In the case of composites containing NOSC, the use of the polymer afforded an increased total pore volume further increased by the addition of CNTs. As observed with the chitosan composites, pore volume and total water sorbed did not correlate in a linear manner. In this case, the increase in total pore volume was associated with a decrease in total water sorbed. This might indicate that pores are formed by the CNTs themselves which contribute to an increase in the hydrophobicity of the surface available for water sorption. The difference in behaviour of the two composites when CNTs are added could be linked to the superior ability of NOSC to disperse efficiently and homogeneuosly CNTs in aqueous environment [28]. 
(a)

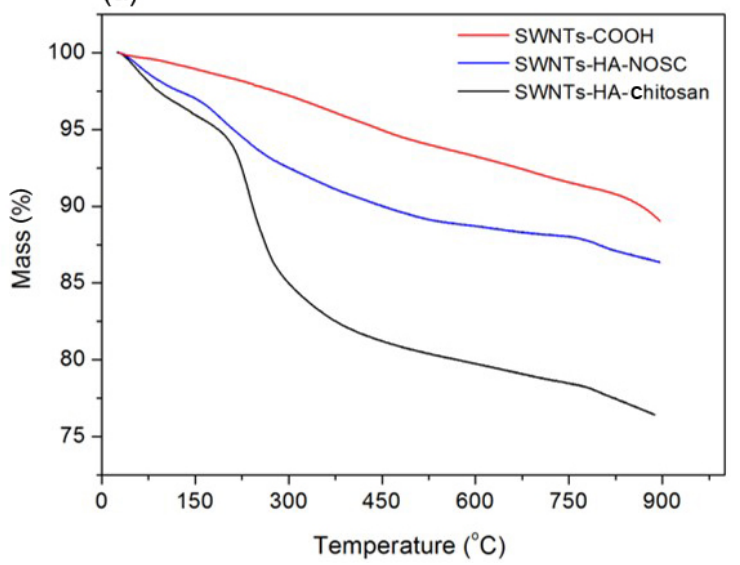

(b)

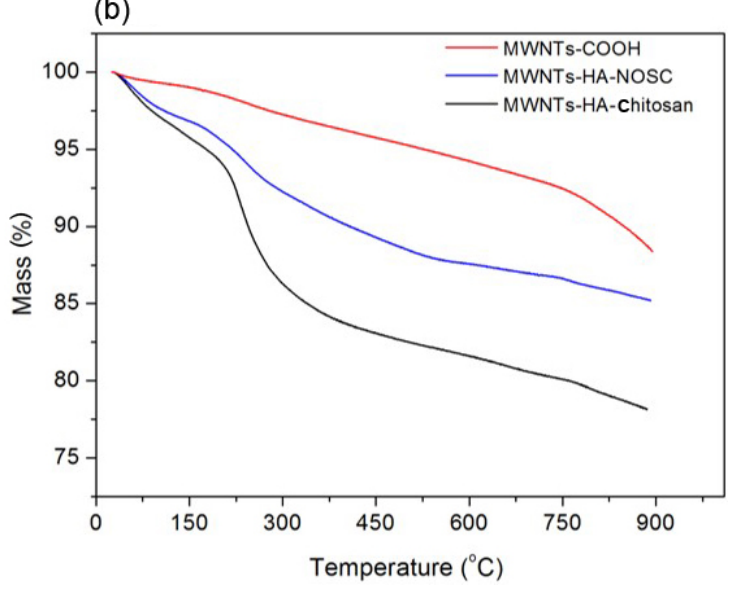

Fig. 5 TGA analysis of (a) SWNTs and (b) MWNTs containing composites.

\section{2 Characterisation of the compressed composite materials}

The HA composites were employed in the formulation of compressed implant materials; the compressed scaffolds contained methylcellulose as a bulking agent, and to improve the compression properties of the powder, additionally methylcellulose should help with the hydration of the scaffold on implantation and favour formation of pores and release for the loaded drug. Furthermore, TCP was added to favour resorption of the composite and it is known that TCP resorbs faster than HA [30]. The compressed formulations were characterised in terms of hardness, friability, disintegration, and drug release properties. All compressed formulations presented a value of hardness between 4 and $7 \mathrm{~N}$ (or 0.8 and $1.4 \mathrm{kPa}$, Fig. 7). The results showed that hardness was mainly affected by the addition of chitosan, as composites containing NOSC did not show a statistically significant difference from the control. In the case of chitosan, the addition of the
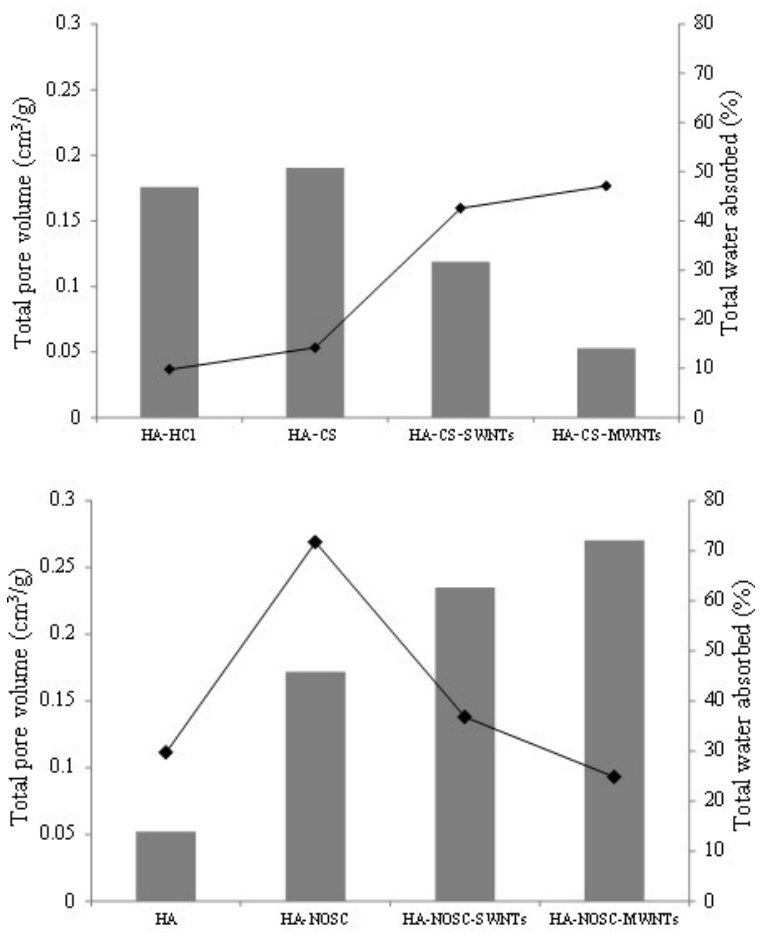

Fig. 6 Total pore volume (grey bars) of HA composite materials and its relation to total water sorbed (black diamonds).

polymer increased significantly the hardness of the material ( $p<0.001$, ANOVA) compared to the control; the increase was similar to that of the composite including both chitosan and SWNTs. However, the addition of MWNTs induced a decrease in hardness to values similar to those of the control $(p>0.05)$. The friability test was carried out to further investigate the properties of the compressed materials. Friability is expressed as the percentage of weight lost by the compressed materials as they undergo shear and friction forces within the drum. Black squares in Fig. 7 show that no material lost more than $5 \%$ weight after 100 cycles of the drum; HA-NOSC-SWNTs leads to the formulation of the least friable implant. In general, it was observed that the addition of CNTs reduced the friability of the implants. The addition of the polymer in the composites gave different results depending on the nature of the polymer itself. Chitosan showed the ability to reinforce the structure, while NOSC, as already seen in the hardness test, did not improve the formulation strength. On the contrary, it afforded the most friable material. The observation of the disintegration pattern of the materials under study, in contact with physiological buffer, can give an indication of the cohesiveness of the material. The results showed that 

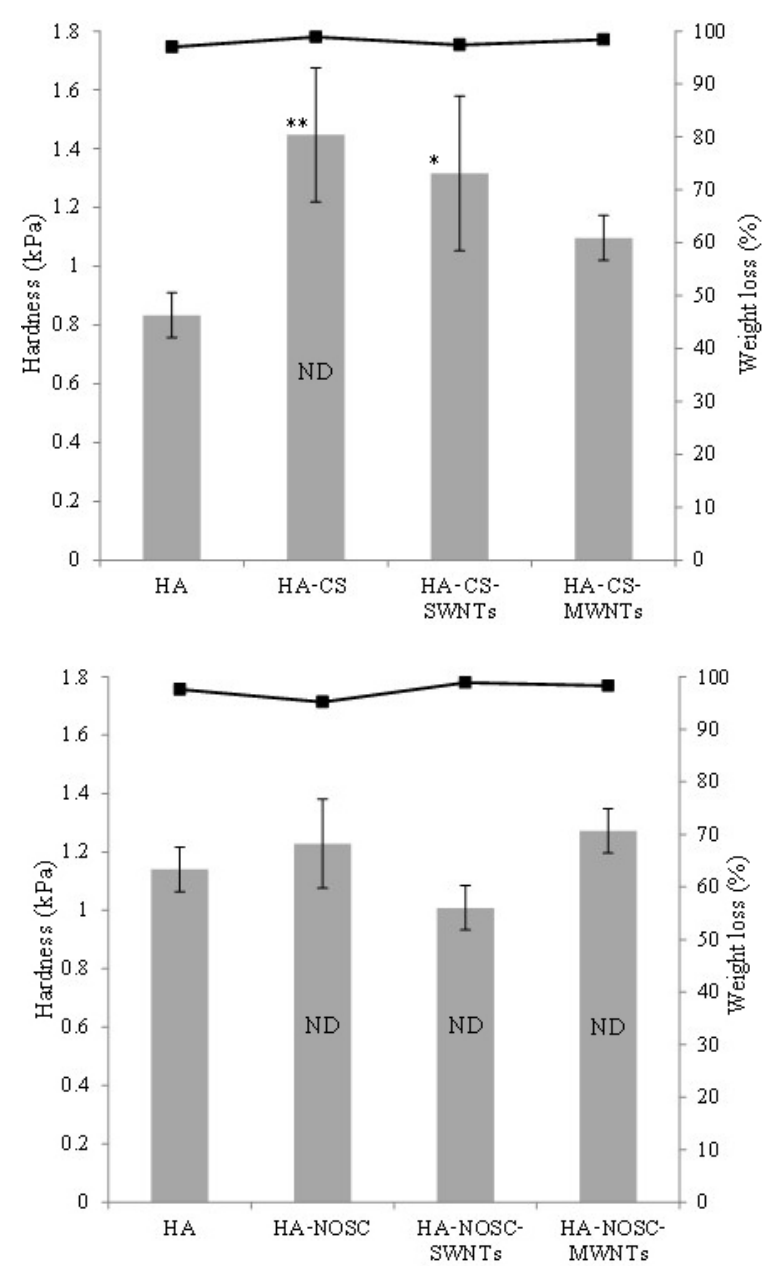

Fig. 7 Correlation between hardness (grey bars) and friability (expressed as percentage of weight loss, black squares) of compressed implant formulations containing either chitosan or NOSC and loaded with ibuprofen sodium as model drug. Hardness results are reported as mean \pm SD $(n=3)$. One way ANOVA $p=0.0034$, Tukey-Kramer post hoc test results are reported in the graph ${ }^{*} p<0.05$ and ${ }^{* *} p<$ 0.01 as compared to the control. ND indicates samples that did not undergo disintegration after $15 \mathrm{~min}$ in PBS at $37{ }^{\circ} \mathrm{C}$.

both control samples started disintegrating after $7 \mathrm{~min}$; however, the addition of both NOSC and chitosan prevented disintegration. With the addition of CNTs, high cohesiveness was observed in composites containing NOSC, while composites containing chitosan disintegrated as the control (SWNTs) or faster (MWNTs). This might be explained by a better interaction between NOSC and CNTs due to hydrophobic interaction between the two materials. Overall, the hardness, friability and disintegration tests gave further insight into the effects that polymers and CNTs have on the mechanical properties of the formulation containing the HA composite materials.

\section{3 Drug release studies}

Release studies of model drugs from the composite formulations were carried out to test the effect of different composition on the ability to control the release profile. Two small molecules of different solubility, ibuprofen and ibuprofen sodium, and a hydrophilic macromolecule (FITC-Dex, molecular weight $40 \mathrm{kDa}$ ) were tested.

As shown in Fig. 8, the addition of polymer, either chitosan or NOSC, to HA led to a slower initial release of ibuprofen. Both polymers seemed to form a diffusion barrier for the drug. The addition of CNTs to the composites reduced the control over the initial release, giving a release profile similar to HA, in the first hour of the study. SWNTs however were able to reduce the overall amount of ibuprofen released over $48 \mathrm{~h}$ when
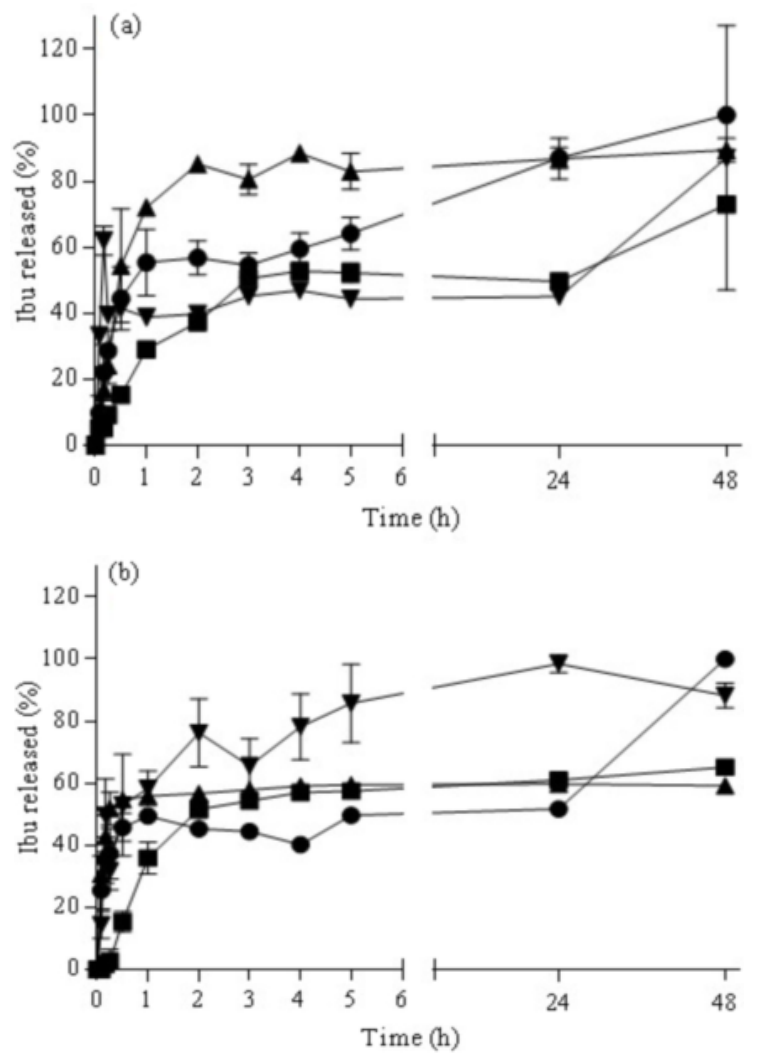

Fig. 8 Release of ibuprofen from HA ( - $)$, HA-polymer ( $-\mathbf{-}$ ), HA-polymer-SWNTs ( $\mathbf{-}$ ), HA-polymer-MWNTs $(\boldsymbol{\nabla})$, where the polymer is chitosan in (a) and NOSC in (b). Data are reported as mean \pm SD $(n=3)$. One way ANOVA for data at $48 \mathrm{~h}$ : chitosan $p<0.0001$, NOSC $p<0.0001$; Dunnet's multicomparison test comparing each set of data with the HA-polymer showed no statistical difference for samples containing chitosan while all samples containing NOSC were significantly different from HA-NOSC $(p<0.05)$. 
used in combination with NOSC $(59.2 \% \pm 0.2 \%, n=3)$. The same was not true for composites containing MWNTs that gave a higher overall release $(109.9 \% \pm 8.6 \%$ and $88.3 \% \pm 4.0 \%$, with chitosan and NOSC respectively); this may be due to a poorer dispersion in the reaction mixture with more tubes interacting with each other and not available for interaction with the drug. The good dispersion ability of NOSC was previously demonstrated in our lab $[16,27,28]$. In general, composites containing NOSC presented a lower amount of ibuprofen released in comparison with the composites containing chitosan; this might be due to hydrophobic interaction between the $\mathrm{C} 8$ chain on the polymer and the drug. When ibuprofen sodium was loaded onto the composite materials (Fig. 9), it was noticed that all composites
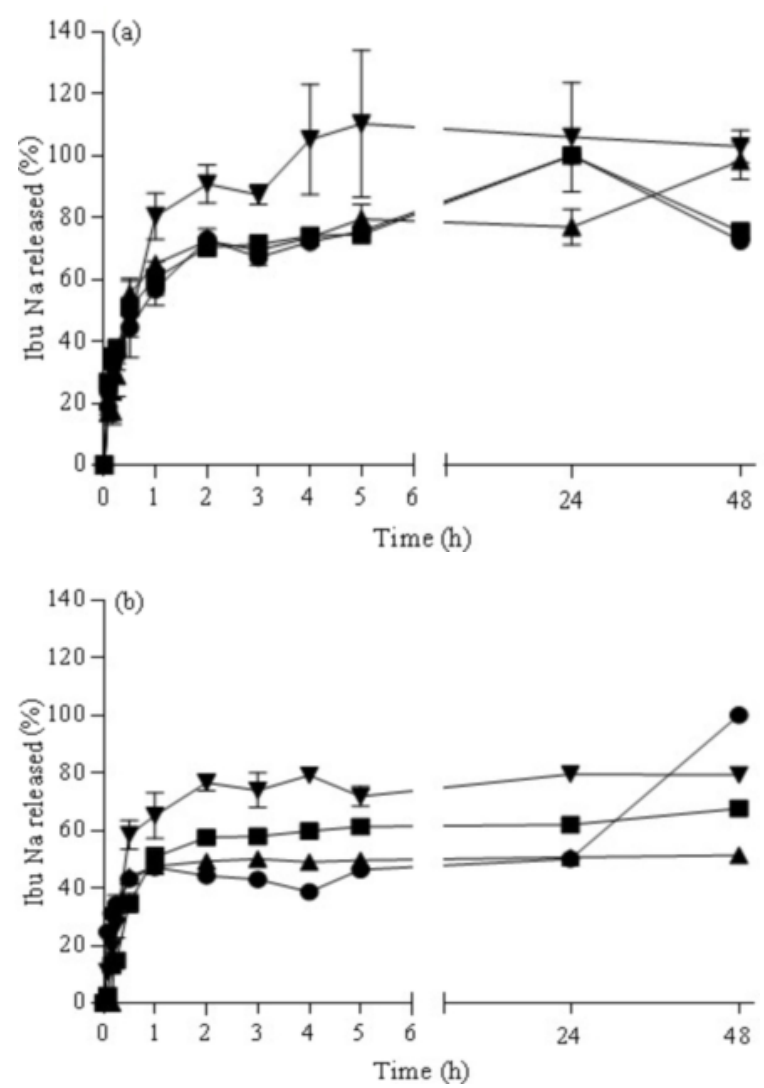

Fig. 9 Release of ibuprofen sodium salt from HA (--), HA-polymer ( $-\boldsymbol{t})$, HA-polymer-SWNTs $(-\mathbf{t})$ ), HA-polymer-MWNTs ( $\nabla-$ ), where the polymer is chitosan in (a) and NOSC in (b). Data are reported as mean $\pm \operatorname{SD}(n=3)$. One way ANOVA for data at $48 \mathrm{~h}$ : chitosan $p<0.0001$, NOSC $p<0.0001$; results of Dunnet's multicomparison test comparing each set of data with the HA-polymer data showed that all samples containing CNTs were statistically different $(p<0.001)$ from the HA-CS samples, while for NOSC all samples were statistically different from HA-NOSC $(p<0.001)$ including HA alone. gave an initial quick release that was not controlled by the addition of the polymers. This might be due to the higher water solubility of the drug. However, overall lower release of the drug was obtained when NOSC and CNTs were used in the composites. Previous studies looking at the effect of chitosan and NOSC on drug release when included in HA composite had demonstrated that the amorphous structure of NOSC compared to the semicrystalline structure of chitosan could be the reason for a slower release from composite containing the amphiphilic chitosan derivative [15].

Release studies were also conducted with FITC-Dex as a high molecular weight model drug. In general, FITC-Dex underwent slower release than ibuprofen and ibuprofen sodium from HA composites containing CNTs, with no composite reaching complete release within $48 \mathrm{~h}$ (Fig. 10). The mechanisms governing FITC-Dex release were assessed by means of the Korsmeyer-Peppas equation.

$$
M_{t} / M_{\infty}=K_{\mathrm{KP}} t^{n}
$$

where $M_{t}$ is the amount of drug released at time $t ; K$ is the apparent rate constant, which is related to the structure and geometry of the dosage form; $M_{t} / M_{\infty}$ is the fractional release of drug at time $t$; and $n$ is the release exponent, the numerical value of which characterises the mechanism of drug release $(n<0.45$, Fickian release; $0.45<n<0.89$, non-Fickian mass transfer; $n>0.89$, case II transport). In all cases, $n>$ 0.89 (Table 5), indicating a case II transport, where the release of the drug is dependent on polymer relaxation, being it swelling or erosion.

In general HA-polymer-CNTs composites containing chitosan showed a slower release in

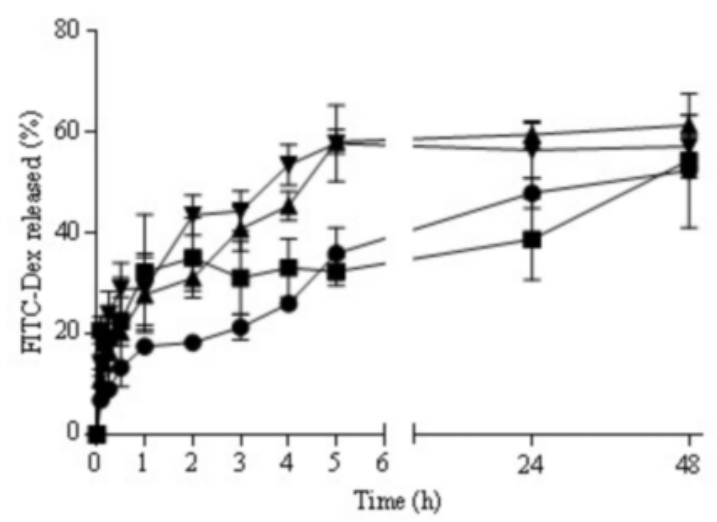

Fig. 10 Release of the FITC-Dex $(40 \mathrm{kDa})$ from HA-CS-SWNTs ( - ) , HA-CS-MWNTs ( - $)$, HA-NOSC-SWNTs $(\boldsymbol{-}-)$, HA-NOSC-MWNTs $(\boldsymbol{\nabla})$. Data are reported as mean $\pm \mathrm{SD}(n=3)$. One way ANOVA for data at $48 \mathrm{~h}, p>0.05$. 
Table 5 Modeling of FITC-Dex release kinetics from HA-polymer-CNTs composites in the first $5 \mathrm{~h}$

\begin{tabular}{lccc}
\hline \multicolumn{1}{c}{ Composite } & $K_{\mathrm{KP}}$ & $n$ & $R^{2}$ \\
\hline HA-NOSC-SWNTs & 0.3736 & 1.6963 & 0.9826 \\
HA-NOSC-MWNTs & 0.3220 & 1.7704 & 0.9715 \\
HA-CS-SWMTs & 0.3654 & 1.6249 & 0.9637 \\
HA-CS-MWNTs & 0.1583 & 1.9934 & 0.8047 \\
\hline
\end{tabular}

comparison with those containing NOSC; this could be attributed to interactions between the thiocyanate and the amino group on chitosan. Furthermore the higher hydrophobicity and higher total pore volume of NOSC containing composites could explain the faster release of the hydrophilic model molecule. However, no statistical difference was appreciated at time point $48 \mathrm{~h}$, indicating that all composites might be good for long term release of high molecular weight drugs.

\section{Conclusions}

HA was successfully prepared by wet precipitation in physiological conditions in the presence of carbon nanotubes with the help of chitosan and its amphiphilic derivative NOSC. The better ability of NOSC to suspend carbon nanotubes in aqueous environment resulted in a more homogeneous dispersion of the CNTs in the composites with an increase in total pore volume and an increase in hydrophobicity of the material. The ease of dispersion of SWNTs in comparison to MWNTs resulted in a better controlled release profile. HA prepared in the presence of NOSC and SWNTs showed potential in the controlled release of both low and high molecular weight hydrophilic drugs.

\section{References}

[1] $\mathrm{Ku} \mathrm{SH}$, Lee M, Park CB. Carbon-based nanomaterials for tissue engineering. Advanced Healthcare Materials 2013, 2: 244-260.

[2] Gao C, Guo Z, Liu J-H, et al. The new age of carbon nanotubes: An updated review of functionalized carbon nanotubes in electrochemical sensors. Nanoscale 2012, 4: 1948-1963.

[3] Roldo M, Fatouros DG. Biomedical applications of carbon nanotubes. Annu Rep Prog Chem, Sect C: Phys Chem 2013, 109: $10-35$.

[4] Tonelli FM, Santos AK, Gomes KN, et al. Carbon nanotube interaction with extracellular matrix proteins producing scaffolds for tissue engineering. Int $J$ Nanomedicine 2012, 7: 4511-4529.

[5] Wang W, Zhu Y, Watari F, et al. Carbon nanotubes/ hydroxyapatite nanocomposites fabricated by spark plasma sintering for bonegraft applications. Appl Surf Sci 2012,
262: 194-199.

[6] Li X, Liu H, Niu X, et al. The use of carbon nanotubes to induce osteogenic differentiation of human adiposederived MSCs in vitro and ectopic bone formation in vivo. Biomaterials 2012, 33: 4818-4827.

[7] Ciapetti G, Granchi D, Devescovi V, et al. Enhancing osteoconduction of PLLA-based nanocomposite scaffolds for bone regeneration using different biomimetic signals to MSCs. Int J Mol Sci 2012, 13: 2439-2458.

[8] Hirata E, Akasaka T, Uo M, et al. Carbon nanotube-coating accelerated cell adhesion and proliferation on poly (L-lactide). Appl Surf Sci 2012, 262: 24-27.

[9] Pan L, Pei X, He R, et al. Multiwall carbon nanotubes/ polycaprolactone composites for bone tissue engineering application. Colloid Surface B 2012, 93: 226-234.

[10] Ormsby R, McNally T, O'Hare P, et al. Fatigue and biocompatibility properties of a poly(methyl methacrylate) bone cement with multi-walled carbon nanotubes. Acta Biomater 2012, 8: 1201-1212.

[11] Roldo M, Fatouros DG. Chitosan-derivative based hydrogels as drug delivery platforms. In Active Implants and Scaffolds for Tissue Regeneration. Zilberman M, Ed. Springer Berlin Heidelberg, 2011: 351-376.

[12] Im O, Li J, Wang H, et al. Biomimetic three-dimensional nanocrystalline hydroxyapatite and magnetically synthesized single-walled carbon nanotube chitosan nanocomposite for bone regeneration. Int J Nanomedicine 2012, 7: 2087-2099

[13] Fonseca-García A, Mota-Morales JD, Quintero-Ortega IA, et al. Effect of doping in carbon nanotubes on the viability of biomimetic chitosan-carbon nanotubes-hydroxyapatite scaffolds. J Biomed Mater Res A 2014, 102: 3341-3351.

[14] Lu XY, Qiu T, Wang XF, et al. Preparation of foam-like carbon nanotubes/hydroxyapatite composite scaffolds with superparamagnetic properties. Appl Surf Sci 2012, 262: 227-230.

[15] Green S, Roldo M, Douroumis D, et al. Chitosan derivatives alter release profiles of model compounds from calcium phosphate implants. Carbohyd Res 2009, 344: 901-907.

[16] Piovesan S, Cox PA, Smith JR, et al. Novel biocompatible chitosan decorated single-walled carbon nanotubes (SWNTs) for biomedical applications: Theoretical and experimental investigations. Phys Chem Chem Phys 2010, 12: $15636-15643$.

[17] Tanase CE, Popa MI, Verestiuc L. Chitosanhydroxyapatite composite obtained by biomimetic method as new bone substitute. In Proceedings of Advanced Technologies for Enhanced Quality of Life, 2009: 42-46.

[18] Tanase CE, Popa MI, Verestiuc L. Biomimetic bone scaffolds based on chitosan and calcium phosphates. Mater Lett 2011, 65: 1681-1683.

[19] Cheng ZH, Yasukawa A, Kandori K, et al. FTIR study on incorporation of $\mathrm{CO}_{2}$ into calcium hydroxyapatite. $J$ Chem Soc, Faraday Trans 1998, 94: 1501-1505.

[20] Danilchenko SN, Kalinkevich OV, Pogorelov MV, et al. Chitosan-hydroxyapatite composite biomaterials made by a one step co-precipitation method: Preparation, 
characterization and in vivo tests. Journal of Biological Physics and Chemistry 2009, 9: 119-126.

[21] Weiner S, Bar-Yosef O. States of preservation of bones from prehistoric sites in the Near East: A survey. J Archaeol Sci 1990, 17: 187-196.

[22] Yasmeen S, Lo MK, Bajracharya S, et al. Injectable scaffolds for bone regeneration. Langmuir 2014, 30: 12977-12985.

[23] Reyes-Gasga J, Martínez-Piñeiro EL, Rodríguez-Álvarez G, et al. XRD and FTIR crystallinity indices in sound human tooth enamel and synthetic hydroxyapatite. Mater Sci Eng C 2013, 33: 4568-4574.

[24] Paul R, Kumbhakar P, Mitra AK. Blue-green luminescence by SWCNT/ZnO hybrid nanostructure synthesized by a simple chemical route. Physica E 2010, 43: 279-284.

[25] Chung S, Kang H, Ocon JD, et al. Enhanced electrical and mass transfer characteristics of acid-treated carbon nanotubes for capacitive deionization. Curr Appl Phys 2015, 15: 1539-1544.

[26] Wang Y, Yang C, Chen X, et al. Biomimetic formation of hydroxyapatite/collagen matrix composite. Adv Eng Mater 2006, 8: 97-100.

[27] Fatouros DG, Power K, Kadir O, et al. Stabilisation of SWNTs by alkyl-sulfate chitosan derivatives of different molecular weight: Towards the preparation of hybrids with anticoagulant properties. Nanoscale 2011, 3: 1218-1224.

[28] Roldo M, Power K, Smith JR, et al. $\mathrm{N}$-Octyl-O-sulfate chitosan stabilises single wall carbon nanotubes in aqueous media and bestows biocompatibility. Nanoscale 2009, 1: 366-373.

[29] Correlo VM, Boesel LF, Bhattacharya M, et al. Hydroxyapatite reinforced chitosan and polyester blends for biomedical applications. Macromol Mater Eng 2005, 290: $1157-1165$.

[30] Bansal S, Chauhan V, Sharma S, et al. Evaluation of hydroxyapatite and beta-tricalcium phosphate mixed with bone marrow aspirate as a bone graft substitute for posterolateral spinal fusion. Indian J Orthop 2009, 43: 234-239.

Open Access The articles published in this journal are distributed under the terms of the Creative Commons Attribution 4.0 International License (http://creativecommons. org/licenses/by/4.0/), which permits unrestricted use, distribution, and reproduction in any medium, provided you give appropriate credit to the original author(s) and the source, provide a link to the Creative Commons license, and indicate if changes were made. 